\section{Optimal target vessel stenosis for radial artery grafting To the Editor:}

The article by Bleiziffer and colleagues ${ }^{1}$ is the latest in a growing body of literature ${ }^{2-6}$ reporting worse radial artery graft patency when anastomosed to coronary targets with moderate disease (eg, 70\%-90\% stenosis) compared with targets with severe stenosis ( $>90 \%$ stenosis). This finding is highly reproducible, as evidenced by analyses of both retrospectively ${ }^{2-5}$ and prospectively $^{1,6}$ collected angiographic data reported by multiple authors at multiple institutions spanning multiple continents. Arguably, the convergence of the published literature to the same target stenosis-radial patency relationship coupled with the available objective mechanistic explanation (competitive flow) for this finding should put this question to rest. More specifically, when one is faced with the choice, radial artery grafts should be preferentially anastomosed to the most severely diseased coronary targets $(>90 \%$ stenosis $)$. These studies, however, have not objectively addressed the question of which conduit type (vein vs radial) should be used for coronary targets with less severe disease (eg, <90\% stenosis). Yet, in their article, Bleiziffer and coworkers concluded the following: "On the basis of our results, we attempt to use the radial artery only for target coronary arteries with $90 \%$ or greater stenosis." I contend that the graft patency data presented to date by these authors and others ${ }^{2-6}$ do not justify such a limitation on radial artery grafting, and that such a position represents a case of over-concluding. More specifically, some (perhaps including the authors ${ }^{1}$ ) may extrapolate this conclusion, which is based only on data for radial artery patency, to suggest that saphenous vein grafts would then be superior to radial artery grafts when placed in coronary targets with less than $90 \%$ disease. I am unaware of any such data. To the contrary, in their most recent report, Desai and colleagues ${ }^{7}$ analyzed prospectively collected radial and vein graft patency data and reported the following: 1) Radial patency increases with increased target stenosis; 2) vein patency does not vary with target stenosis; and, most important, 3) radial patency is superior to that of vein patency when placed in both moderately and severely diseased coronary targets. These data indicate that the radial artery should be preferred to the vein even for

moderately diseased coronary targets, a conclusion that contradicts the authors' recommendation in this article. ${ }^{1}$

\section{Robert H. Habib, PhD \\ Cardiovascular and Pulmonary Research Yvonne Viens, SGM, Research Institute St Vincent Mercy Medical Center Toledo, Ohio \\ Department of Surgery University of Toledo College of Medicine Toledo, Ohio}

\section{References}

1. Bleiziffer S, Hettich I, Eisenhauer B, Ruzicka D, Wottke M, Hausleiter J, et al. Patency rates of endoscopically harvested radial arteries one year after coronary artery bypass grafting. J Thorac Cardiovasc Surg. 2007; 134:649-56.

2. Royse AG, Royse CF, Tatoulis J, Grigg LE, Shah $\mathrm{P}$, Hunt D, et al. Postoperative radial artery angiography for coronary artery bypass surgery. Eur J Cardiothorac Surg. 2000;17: 294-304.

3. Maniar HS, Sundt TM, Barner HB, Prasad SM, Peterson L, Absi T, et al. Effect of target stenosis and location on radial artery graft patency. J Thorac Cardiovasc Surg. 2002;123:45-52.

4. Maniar HS, Barner HB, Bailey MS, Prasad SM, Moon MR, Pasque MK, et al. Radial artery patency: are aortocoronary conduits superior to composite grafting? Ann Thorac Surg. 2003;76:1498-504.

5. Desai ND, Cohen EA, Naylor CD, Fremes SE. A randomized comparison of radial-artery and saphenous-vein coronary bypass grafts. $N$ Engl J Med. 2004;351:2302-9.

6. Lemma M, Mangini A, Gelpi G, Innorta A, Spina A, Antona C. Is it better to use the radial artery as a composite graft? Clinical and angiographic results of aorto-coronary versus Y-graft. Eur J Cardiothorac Surg. 2004;26: 110-7.

7. Desai ND, Naylor CD, Kiss A, Cohen EA, Feder-Elituv R, Miwa S, et al. Impact of patient and target-vessel characteristics on arterial and venous bypass graft patency: insight from a randomized trial. Circulation. 2007; 115:684-91.

doi:10.1016/j.jtcvs.2007.10.017

\section{Reply to the Editor:}

We fully agree with Habib that, on the basis of the current literature, radial artery patency is supposed to be superior to sapheneous vein patency even when the grafts are anastomosed to moderately stenosed coronary targets. Nevertheless, we believe that the radial artery as a graft for coronary artery bypass grafting should be used under the most promising conditions, that is, for highly stenosed coronary vessels. Radial artery harvesting is more complex than vein harvesting and may lead to more serious complications, such as circulatory or sensory disturbances at the donor arm. Considering this in a risk-benefit analysis, we argue that the radial artery should be harvested only when optimum patency rates are expected for the individual patient. Therefore, we adhere to our recommendation to place the radial artery only to highly stenosed target vessels.

Sabine Bleiziffer, $M D$ Ruediger Lange, $P h D, M D$ Clinic for Cardiovascular Surgery German Heart Center Munich Munich, Germany doi:10.1016/j.jtcvs.2007.10.016

\section{Prolonged mechanical ventilation after cardiovascular surgery \\ To the Editor:}

I read with great interest the excellent article by Dr Murthy and colleagues ${ }^{1}$ describing prolonged ventilatory dependence after cardiovascular surgery. The following clinical questions have arisen since my review of this study:

1. What are the possible explanations for the significant decrease in ventilatory dependence over time? What major changes in delivery of care occurred during the study period (1998-2001)?

2. What was aprotinin exposure in the study cohort? What are the possible ways aprotinin exposure affected the study results? Aprotinin has been associated with a tendency to reduced ventilation time and reduced pulmonary complications after thoracic aortic surgery. ${ }^{2}$ Furthermore, in meta-analysis, aprotinin was the only antifibrinolytic associated with a significantly decreased rate of reoperation for bleeding. ${ }^{3}$ Both aortic surgery and reoperation for bleeding were significant predictors for ventilatory dependence in your published study.

3. Were there any major changes in anesthetic techniques during the study period that may have affected the incidence of ventilatory dependence? 
I congratulate the authors again on a most thought-provoking study. I look forward to their feedback about these considerations.

John G. T. Augoustides, MD, FASE Department of Anesthesiology and Critical Care Hospital of the University of Pennsylvania Philadelphia, PA 19104-4283

Financial support: Department of Anesthesiology and Critical Care, Hospital of the University of Pennsylvania.

\section{References}

1. Murthy SC, Arroliga AC, Walts PA, Feng J, Yared JP, Lytle B, et al. Ventilatory dependence after cardiovascular surgery. $J$ Thorac Cardiovasc Surg. 2007;134:484-90.

2. Sedrakyan A, Wu A, Sedrakyan G, DienerWest M, Tranquilli M, Elefteriades J. Aprotinin use in thoracic aortic surgery: safety and outcomes. J Thorac Cardiovasc Surg. 2006; 132:909-17.

3. Brown JR, Birkmeyer NJO, O'Connor GT. Meta-analysis comparing the effectiveness and adverse outcomes of antifibrinolytic agents in cardiac surgery. Circulation. 2007; 115:2801-13.

doi:10.1016/j.jtcvs.2007.09.056

\section{Reply to the Editor:}

We appreciate the comments provided by Dr Augoustides in regard to our recent article "Ventilatory Dependency After Cardiovascular Surgery." Our article attempts to define the risks for ventilator dependency and determine the outcomes of patients who develop this problem after their index cardiovascular surgery. Two inquiries were made (as points [a] and [c] are closely related).

We were surprised ourselves to find that there has been a continuing trend toward a lower prevalence of ventilator dependency at our institute. A declining trend was noted in previous studies from our institute published several years before. That this trend has continued through the current study has both thrilled and perplexed us. It was impossible for us to determine which quantifiable variables might be responsible for this trend because of a variety of confounding factors, not the least of which is time itself. What is even more interesting is that this phenomenon has occurred in the setting of increasing complexity and acuity of illness. It is our suspicion that several coincident changes might be responsible. There has been a steady improvement in myocardial protection over the years and a progressive increase of intraoperative echocardiography use; these factors likely promote more rapid myocardial recovery. Less narcotics are being given intraoperatively, and this may facilitate earlier separation from mechanical ventilatory support. In addition, there has been a noticeable increase in intensivist staffing, such that there is seemingly more planning and continuity of care in the intensive care unit, which again, might favorably affect weaning.

Second, aprotinin is used sparingly at our institution (in $<1 \%$ of patients). This is, in part, secondary to cost, but also for concerns regarding thrombotic complication and renal dysfunction, all without clearly demonstrable benefit. There are 2 populations for whom we do use aprotinin on more occasions: patients with ventricular-assist devices and patients undergoing a second cardiac transplant. These groups were excluded from our study.

Sudish C. Murthy, MD, PhD Thoracic and Cardiovascular Surgery The Cleveland Clinic Cleveland, Ohio doi:10.1016/j.jtcvs.2007.10.025

\section{Prosthesis-patient mismatch after mitral valve replacement: Back to reality \\ To the Editor:}

We read with interest the article of Totaro and Argano. ${ }^{1}$ We have, however, several concerns with regard to the validity of the results and conclusions presented in this article. First, the title of the article, "Patient-Prosthesis Mismatch after Mitral Valve Replacement: Myth or Reality?" is inappropriate. Mitral prosthesis-patient mismatch (PPM) is equivalent to a residual mitral stenosis, related to the fact that most prosthetic valves have a hemodynamic performance, and thus a valve effective orifice area (EOA), that is inferior to that of the normal native valve. Thus concluding that PPM is a myth would be equivalent to saying that mitral stenosis (or aortic stenosis for aortic PPM) does not exist, and that this is a benign phenomenon, which is of course not the case. In this regard, several studies have demonstrated that PPM is a frequent hemodynamic phenomenon after mitral or aortic valve replacement. ${ }^{2-5}$ The important question is rather to determine the impact of PPM on the hemodynamic, functional, and clinical outcomes, and at which degree of severity and in which categories of patients this impact of PPM becomes statistically significant and clinically relevant. Unfortunately, the data provided by Totaro and Argano $^{1}$ do not permit an answer to these important questions.

There are serious concerns about the validity of the Doppler echocardiographic data, and especially of those of the valve EOA. This is a crucial aspect, because the identification and quantification of PPM are based on these data. First, it is intriguing to see that the EOAs measured in vivo by Doppler echocardiography, especially for the 25- and 27-mm valves, were larger than the EOAs measured in vitro by the manufacturer. A recent study has indeed demonstrated that, as opposed to the observation in this study, the in vitro EOAs provided by the manufacturer grossly overestimate the in vivo EOAs and are thus not valid for prediction of PPM. ${ }^{6}$ Totaro and Argano also used the label prosthesis size as a surrogate for PPM, whereas previous studies have shown that this parameter is not valid for identification of PPM and prediction of its hemodynamic and clinical consequences. ${ }^{3,6,7}$

Moreover, the huge variability in the EOA measurements for a given prosthesis size (from 1.0 to $4.9 \mathrm{~cm}^{2}$ for the 29 -mm prostheses!), the complete absence of correlation between the EOA and the transprosthetic gradient, and the recording of high transprosthetic gradients $(>15 \mathrm{~mm} \mathrm{Hg})$ in several patients despite the calculation of large EOAs and indexed EOAs (see Figure 3 of the article $^{1}$ ) further support the concerns regarding the validity of the EOA measurements and thus the identification of PPM. The presence of gradients greater than 15 $\mathrm{mm} \mathrm{Hg}$ in the mitral position definitely cannot be considered as "favorable hemodynamics," as concluded by Totaro and Argano. ${ }^{1}$ In fine, these observations suggest that a large proportion of the patients included in this series were misclassified with respect to the presence or absence of PPM.

Totaro and Argano only measured the valve hemodynamics and systolic pulmonary arterial pressure at predischarge examination or at 30 days. It is well known that the measurements of valve EOA, transvalvular gradients, and pulmonary pressure in the early postoperative period are often unreliable because of the poor acoustic window, hyperdynamic state, or flow acceleration in the left ventricular outflow tract. 